\title{
Adaptive-Neural-Network-Based Shape Control for a Swarm of Robots
}

\author{
Xuejing Lan $\mathbb{D},{ }^{1,2}$ Zhenghao $W u\left(\mathbb{D},,^{1}\right.$ Wenbiao Xu $\mathbb{D},^{3}$ and Guiyun Liu $\mathbb{D}^{1,4}$ \\ ${ }^{1}$ School of Mechanical and Electrical Engineering, Guangzhou University, Guangzhou 510006, China \\ ${ }^{2}$ MOE Key Laboratory of Image Processing and Intelligence Control, Wuhan 430074, China \\ ${ }^{3}$ Guangdong Institute of Metrology, Guangzhou 510405, China \\ ${ }^{4}$ Center for Intelligent Equipment and Internet-Connected Systems, China
}

Correspondence should be addressed to Wenbiao Xu; xwb8911@hust.edu.cn

Received 9 July 2018; Accepted 19 September 2018; Published 2 December 2018

Guest Editor: Andy Annamalai

Copyright (c) 2018 Xuejing Lan et al. This is an open access article distributed under the Creative Commons Attribution License, which permits unrestricted use, distribution, and reproduction in any medium, provided the original work is properly cited.

This paper considers the region-based formation control for a swarm of robots with unknown nonlinear dynamics and disturbances. An adaptive neural network is designed to approximate the unknown nonlinear dynamics, and the desired formation shape is achieved by designing appropriate potential functions. Moreover, the collision avoidance, velocity consensus, and region tracking are all considered in the controller. The stability of the multirobot system has been demonstrated based on the Lyapunov theorem. Finally, three numerical simulations show the effectiveness of the proposed formation control scheme to deal with the narrow space, loss of robots, and formation merging problems.

\section{Introduction}

Formation control problem of a multiagent system has long been the concern of many scholars, as it is one of the important problems in the cooperative control and has broad applications, such as in mobile robots, ships, submarines, and flight vehicles [1-5]. There exist many formation control strategies that are categorized as position-based, displacement-based, and distance-based formation control schemes according to the different requirements on the sensing capability and the interaction topology [6-10].

However, most of the existing works focus on the leaderfollower network and the graph theoretic method. In these formation constructions, the geometric relationship between robots is usually fixed with given position, angle, placement, or distance [11-14]. With the increasing number of robots in the formation, it is difficult to accurately locate each robot, and the constraint relationship among the robots will become more complicated. Therefore, it is challenging for the existing formation control strategies to deal with the large swarm of robots.
The region-based formation control is a complement to the above formation control methods that can solve the formation control problem for the large swarm of robots $[15,16]$. The swarm of robots are required to stay inside the desired region or on the edge of the region. It focuses more on the shape of the whole formation, rather than on the inner sensing capability and interaction topology. Unlike the traditional formation constructions, the geometric relationship between robots in the region-based formation control is not fixed and can change constantly during the motion. Therefore, complicated formation shape can be achieved by designing appropriate regional boundary conditions, such as circular shape, ring shape, and crescent shape [17]. Jung and Kim proposed a potential-function-based shape formation control law, where a diamond-shaped formation and a heart-shaped formation are achieved [18]. There is no special leader or follower in these region-based formations where the rolls and identities of the robots are the same. Haghighi and Cheah achieved a plane-shape formation, but a leading group and some follower groups were introduced in the formation control scheme with a global 
leader in the leading group and a local leader in each follower group [19]. Hou and Cheah achieved a car-shape formation and an airplane-shape formation for the swarm of robots based on various basic shapes [20].

The region-based formation control also has the advantage to deal with the obstacle avoidance problems, especially passing the narrow spaces. Hou et al. introduced a scaling matrix to change the size of the desired region and a rotation matrix to rotate the desired region for passing through a tunnel [21]. Because the shape of the formation is flexible and variable, there is a chance to settle the problem of passing a narrow space by changing the shape of the desired region directly. In addition, it is complicated to solve the formation reconstruction and formation merging problems by the traditional distance-based formation control schemes [22-24]. The region-based formation control scheme provides a new way to deal with these problems.

In this paper, we focus on the region-based formation control for a swarm of robots with unknown nonlinear dynamics and disturbances. The proposed controller is formulated into three parts: region-based shape realization, collision avoidance, and velocity synchronization. The potential function of robot is designed based on the shape objective functions for region-based shape realization, and the artificial potential field (APF) between each pair of neighboring robots is defined for collision avoidance. Moreover, the local motion synchronization error and region tracking error are both controlled for velocity consensus. Considering the excellent estimation ability of a neural network [25-27], we approximate the unknown nonlinear dynamics of the robots by an adaptive neural network. The stability of the multirobot system has been demonstrated based on the Lyapunov theorem, and three numerical simulations are performed. A narrow space is passed by the swarm of robots with the shape of the formation changed, without the need of a scaling matrix or a rotation matrix. When some robots failed due to some accidents, the remaining robots reconstruct the formation adaptively. In addition, two moving formations are merged together successfully.

The outline of this paper is listed as follows. Background is introduced in Section 2. The procedure of formation controller design is presented in Section 3. Numerical simulations are shown in Section 4, and a conclusion is in Section 5.

\section{Background}

It is assumed that each robot $R_{i}$ can measure the relative positions of its neighboring robots via an onboard sensor within its detection range. The state of the robot will be represented with respect to a global coordinate system ${ }^{g} \sum$ in this paper. The position and the velocity of robot $R_{i}$ at time $t$ are denoted by $p_{i}(t)=\left[p_{i x}, p_{i y}\right]^{T}$ and $v_{i}(t)=\left[v_{i x}, v_{i y}\right]^{T}$, respectively. The dynamics of the robot $R_{i}$ can be modeled as

$$
\begin{aligned}
& \dot{p}_{i}(t)=v_{i}(t), \\
& \dot{v}_{i}(t)=f_{i}(t)+u_{i}(t)+w_{i}(t),
\end{aligned}
$$

where $u_{i}$ is the control input of robot $R_{i}$. $w_{i}$ represents the disturbances from the environment satisfying $\left|w_{i}\right| \leq w_{M}$, where $w_{M}$ is a positive constant. $f_{i}$ is the unknown nonlinear dynamics. $i=1, \ldots, N$, and $N$ is the total number of the swarm of robots.

\section{Formation Controller Design}

3.1. Region-Based Shape Realization. A moving region is defined for the swarm of robots to stay inside, which can be formed as various shapes by designing appropriate objective functions. The global shape objective function of robot $R_{i}$ is defined as follows:

$$
g\left(p_{i}, p_{r}\right)=\left[g_{1}\left(p_{i}, p_{r}\right), \ldots, g_{M}\left(p_{i}, p_{r}\right)\right] \leq 0,
$$

where $M$ is the total number of the objective functions that the robots should satisfy. $p_{r}$ denotes the position of the reference point in the desired region and satisfies the following condition:

$$
\begin{aligned}
& \dot{p}_{r}=v_{r}, \\
& \dot{v}_{r}=a_{r},
\end{aligned}
$$

where $v_{r}$ is the velocity of the reference point. $a_{r}$ is the acceleration of the reference point satisfying $\left|a_{r}\right| \leq a_{M}$, where $a_{M}$ is a positive constant. It is assumed that the position and the velocity of the reference point in the desired region are known by the robots. It should be noted that each of the shape objective functions $g_{m} \leq 0$ when the robot stays inside the desired region, otherwise, $g_{m}>0$. Thus, the potential function of robot $R_{i}$ based on the shape objective functions can be presented by

$$
G_{i}=\sum_{m=1}^{M} \frac{1}{2}\left[\max \left(0, g_{m}\left(p_{i}, p_{r}\right)\right)\right]^{2} .
$$

In (5), the term max $\left(0, g_{m}\left(p_{i}, p_{r}\right)\right)$ will be zero if $g_{m}$ $\leq 0$, which means that the corresponding potential function is zero if the shape objective function is satisfied. Then, we can get the gradients of the potential function for the robot $R_{i}$ as follows:

$$
\nabla_{i} G_{i}=\sum_{m=1}^{M} \max \left(0, g_{m}\left(p_{i}, p_{r}\right)\right)\left(\nabla_{i} g_{m}\left(p_{i}, p_{r}\right)\right)
$$

Then, the controller of robot $R_{i}$ for region-based shape realization can be represented as

$$
u_{i}^{s}=-k_{g} \nabla_{i} G_{i}
$$

where $k_{g}$ is a positive constant.

Remark 1. Various shapes can be formed by designing appropriate objective functions. For example, the objective functions for a ring shape can be designed as 


$$
\begin{aligned}
& g_{1}\left(p_{i}, p_{r}\right)=r_{1}^{2}-\left\|p_{i}-p_{r}\right\|^{2} \leq 0, \\
& g_{2}\left(p_{i}, p_{r}\right)=\left\|p_{i}-p_{r}\right\|^{2}-r_{2}^{2} \leq 0,
\end{aligned}
$$

where $r_{1}$ and $r_{2}$ are the parameters of the ring shape satisfying $r_{1}<r_{2}$.

3.2. Collision Avoidance. The detection range of robot $R_{i}$ denoted as $d$ is larger than the allowable minimum distance between the robots and can be adjusted according to different target formation adaptively. Then, the neighboring set of robot $R_{i}$ is defined as $\mathcal{N}_{i}=\left\{j \in[1, N] \mid\left\|p_{i}-p_{j}\right\| \leq d\right\}$. It should be noted that the neighboring set $\mathcal{N}_{i}$ is variable during the moving of the robots. In order to achieve the shape control without collisions, a potential function between each pair of robots is defined as follows.

$$
\psi_{i j}=\left\{\begin{array}{l}
\frac{c}{\left\|p_{i}-p_{j}\right\|^{2}}, \quad j \in \mathcal{N}_{i}, \\
0, \quad j \notin \mathscr{N}_{i},
\end{array}\right.
$$

where $c$ is a positive constant. $\|\cdot\|$ denotes the Euclidean norm. The potential for the robot $R_{i}$ is defined as the sum of all APF associated with every one of its neighbors.

$$
\Psi_{i}=\sum_{j \in \mathcal{N}_{i}} \psi_{i j}
$$

Then, we can get the gradients of the APF for the robot $R_{i}$ as follows:

$$
\nabla_{i} \Psi_{i}=\sum_{j \in \mathcal{N}_{i}} \nabla_{i} \psi_{i j}
$$

Thus, the controller of robot $R_{i}$ for collision avoidance can be represented as follows:

$$
u_{i}^{c}=-k_{q} \nabla_{i} \Psi_{i}
$$

where $k_{q}$ is a positive constant.

3.3. Velocity Synchronization. The local motion synchronization error of robot $R_{i}$ is defined as

$$
e_{i v}=\sum_{j \in \mathcal{N}_{i}}\left(v_{i}-v_{j}\right)
$$

The region tracking error of robot $R_{i}$ is defined as

$$
e_{i v r}=v_{i}-v_{r} .
$$

Then, the controller of robot $R_{i}$ for velocity consensus can be designed as follows:

$$
u_{i}^{v}=-k_{v}\left(e_{i v}+\beta e_{i v r}\right) \triangleq-k_{v} e_{\mathrm{is}},
$$

where $k_{v}$ and $\beta$ are positive constants. The error vector of the swarm robots can be represented as follows:

$$
e_{s}=\left(L+\beta I_{N}\right)\left(v-1 \otimes v_{r}\right)
$$

where $e_{s}=\left[e_{1 s}, \ldots, e_{N s}\right]^{T}$ and $v=\left[v_{1}, \ldots, v_{N}\right]^{T} . I_{N}$ denotes an identity matrix of dimension $N . \mathbf{1}=[1, \ldots, 1]^{T} \in \mathbb{R}^{N}$, and $\otimes$ denotes the Kronecker product. Define $a_{i j}=1$, if $j \in$ $\mathcal{N}_{i}$; otherwise, $a_{i j}=0$. The Laplacian matrix $L=D-A$. $D=$ $\operatorname{diag}\left\{d_{1}, \ldots, d_{N}\right\}^{T}$ with $d_{i}=\sum_{j \in N_{i}} a_{i j}$, and $A=\left[a_{i j}\right]^{N \times N}$.

Taking the time derivative of $e_{s}$, we have

$$
\dot{e}_{s}=\left(L+\beta I_{N}\right)\left[f+u+w-\mathbf{1} \otimes a_{r}\right],
$$

where $f=\left[f_{1}, \ldots, f_{N}\right]^{T} \cdot u=\left[u_{1}, \ldots, u_{N}\right]^{T} \cdot w=\left[w_{1}, \ldots, w_{N}\right]^{T}$. The unknown $f_{i}(t)$ can be approximated by a radial basis function neural network (RBFNN) as follows:

$$
f_{i}(t)=\Phi_{i}^{T} \phi\left(\mu_{i}\right)+\varepsilon_{i}, \quad i=1, \ldots, N,
$$

where $\Phi_{i}^{T} \in \mathbb{R}^{n}$ represents a set of RBFNN coefficients. $\mu_{i}=$ $\left[e_{i v}, e_{i v r}\right]^{T} . \varepsilon_{i}$ is the approximation error satisfying $\left|\varepsilon_{i}\right| \leq \varepsilon_{M}$, where $\varepsilon_{M}$ is a positive constant. $\phi\left(\mu_{i}\right) \in \mathbb{R}^{n}$ is a valued function vector based on the Gaussian function $\phi_{k}\left(\mu_{i}\right)$ chosen as follows:

$$
\phi_{k}\left(\mu_{i}\right)=\exp \left(-\frac{\left\|\mu_{i}-\varsigma_{k}\right\|^{2}}{\eta^{2}}\right), \quad k=1, \ldots, n,
$$

where $\varsigma_{k} \in \mathbb{R}^{2}$ is a constant vector denoting the center of the basis function. $\eta$ is a constant denoting the width of the basis function. Note that the basic function $\phi_{k}\left(\mu_{i}\right)$ is bounded as $\left|\phi_{k}\left(\mu_{i}\right)\right| \leq \phi_{M}$, where $\phi_{M}$ is a positive constant.

3.4. Control Law. The final control law of robot $R_{i}$ is designed as follows:

$$
u_{i}(t)=-k_{v} e_{\mathrm{is}}-\widehat{\Phi}_{i}^{T} \phi\left(\mu_{i}\right)-k_{g} \nabla_{i} G_{i}-k_{q} \nabla_{i} \Psi_{i}
$$

where $\widehat{\Phi}_{I}^{T} \phi\left(\mu_{i}\right)$ is used to estimate $f_{i}(t) . \widehat{\Phi}_{i}$ is a vector of RBFNN weights with the adaptive updating law proposed as

$$
\dot{\widehat{\Phi}}_{i}=\gamma\left[\phi\left(\mu_{i}\right) e_{\mathrm{is}}\left(d_{i}+\beta\right)-k_{F} \widehat{\Phi}_{i}\right]
$$

where $\gamma$ and $k_{F}$ are positive constants. Then, the error dynamics in (17) become

$$
\begin{aligned}
\dot{e}_{s}= & \left(L+\beta I_{N}\right)\left[-\tilde{\Phi}^{T} \phi(\mu)+\varepsilon-k_{v} e_{s}\right. \\
& \left.-k_{g} \nabla G-k_{q} \nabla \Psi+w-\mathbf{1} \otimes a_{r}\right],
\end{aligned}
$$

where $\varepsilon=\left[\varepsilon_{1}, \ldots, \varepsilon_{N}\right]^{T}, \phi(\mu)=\left[\phi^{T}\left(\mu_{1}\right), \ldots, \phi^{T}\left(\mu_{N}\right)\right]^{T}, \widehat{\Phi}^{T}=$ $\operatorname{diag}\left\{\widehat{\Phi}_{1}^{T}, \ldots, \widehat{\Phi}_{N}^{T}\right\}, \Phi^{T}=\operatorname{diag}\left\{\Phi_{1}^{T}, \ldots, \Phi_{N}^{T}\right\}$, and $\tilde{\Phi}=\widehat{\Phi}-$ $\Phi . \Psi=\left[\Psi_{1}, \ldots, \Psi_{N}\right]^{T} . G=\left[G_{1}, \ldots, G_{N}\right]^{T}$. The main result of this paper is given by the following theorem. 
Theorem 1. Consider a swarm of robots with dynamics in (1) and (2). Select the control law in (20) and the adaptive law in (21). Then, there exist positive constants $k_{v}, \beta, \gamma, k_{F}, k_{g}$, and $k_{q}$, such that all the robots stay inside a moving region of specific shape, and the local motion synchronization errors converge to a small neighborhood of the origin.

Proof. Then, we consider a Lyapunov function:

$$
V=\frac{1}{2} e_{s}^{T} e_{s}+\frac{1}{2} \operatorname{tr}\left\{\tilde{\Phi}^{T} \Gamma^{-1} \tilde{\Phi}\right\}
$$

where $\Gamma=I_{N} \otimes \gamma I_{n} . I_{n}$ denotes an identity matrix of dimension $n$. Taking the time derivative of $V$ and substituting (21) and (22) into it, we can get

$$
\begin{aligned}
\dot{V}= & e_{s}^{T}\left(L+\beta I_{N}\right)\left[-\tilde{\Phi}^{T} \phi(\mu)+\varepsilon-k_{v} e_{s}\right. \\
& \left.-k_{g} \nabla G-k_{q} \nabla \Psi+w-1 \otimes a_{r}\right] \\
& +\operatorname{tr}\left\{\tilde{\Phi}^{T} \Gamma^{-1}\left[\Gamma\left(\phi(\mu) e_{s}^{T}\left(D+\beta I_{N}\right)-k_{F} \widehat{\Phi}\right)\right]\right\} \\
= & e_{s}^{T}\left(L+\beta I_{N}\right)\left(\varepsilon+w-\mathbf{1} \otimes a_{r}-k_{g} \nabla G-k_{q} \nabla \Psi\right) \\
& -e_{s}^{T} k_{v}\left(L+\beta I_{N}\right) e_{s}-e_{s}^{T}\left(L+\beta I_{N}\right) \tilde{\Phi}^{T} \phi(\mu) \\
& +\operatorname{tr}\left\{\tilde{\Phi}^{T}\left[\phi(\mu) e_{s}^{T}\left(D+\beta I_{N}\right)-k_{F} \widehat{\Phi}\right]\right\} \\
= & e_{s}^{T}\left(L+\beta I_{N}\right)\left(\varepsilon+w-\mathbf{1} \otimes a_{r}-k_{g} \nabla G-k_{q} \nabla \Psi\right) \\
& -e_{s}^{T} k_{v}\left(L+\beta I_{N}\right) e_{s}+\operatorname{tr}\left\{\tilde{\Phi}^{T} \phi(\mu) e_{s}^{T}\left(D+\beta I_{N}\right)\right. \\
& \left.-\tilde{\Phi}^{T} k_{F} \widehat{\Phi}-\tilde{\Phi}^{T} \phi(\mu) e_{s}^{T}\left(L+\beta I_{N}\right)\right\} \\
= & e_{s}^{T}\left(L+\beta I_{N}\right)\left(\varepsilon+w-\mathbf{1} \otimes a_{r}-k_{g} \nabla G-k_{q} \nabla \Psi\right) \\
& -e_{s}^{T} k_{v}\left(L+\beta I_{N}\right) e_{s}+\operatorname{tr}\left\{\tilde{\Phi}^{T} \phi(\mu) e_{s}^{T} A\right\}-k_{F} \operatorname{tr}\left\{\tilde{\Phi}^{T} \widehat{\Phi}\right\} .
\end{aligned}
$$

Then, we can obtain

$$
\begin{gathered}
\dot{V} \leq\left\|e_{s}\right\| \bar{\rho}\left(L+\beta I_{N}\right) \Theta_{M}+\|\tilde{\Phi}\|_{F} \phi_{M}\left\|e_{s}\right\| \bar{\rho}(A) \\
-k_{v} \underline{\rho}\left(L+\beta I_{N}\right)\left\|e_{s}\right\|^{2}-k_{F} \operatorname{tr}\left\{\tilde{\Phi}^{T} \widehat{\Phi}\right\},
\end{gathered}
$$

where $\bar{\rho}(\cdot)$ and $\rho(\cdot)$ denote the maximum and minimum singular values of a matrix, respectively. $\|\cdot\|_{F}$ denotes the Frobenius norm. $\Theta_{M}=\varepsilon_{M}+w_{M}+a_{M} \sqrt{N}+k_{g} \varsigma_{G M}+k_{q} \varsigma_{\Psi M}$, with $\varsigma_{G M}$ and $\varsigma_{\Psi M}$ representing the magnitude of the maximum potential force of $\nabla G$ and $\nabla \Psi$, respectively. Using Young's inequality, we obtain

$$
-k_{F} \operatorname{tr}\left\{\tilde{\Phi}^{T} \widehat{\Phi}\right\} \leq-\frac{k_{F}}{2}\|\tilde{\Phi}\|_{F}^{2}+\frac{k_{F}}{2}\|\Phi\|_{F}^{2}
$$

Then, (25) can be represented as

$$
\begin{aligned}
\dot{V} \leq & \left\|e_{s}\right\| \bar{\rho}\left(L+\beta I_{N}\right) \Theta_{M}+\|\tilde{\Phi}\|_{F} \phi_{M}\left\|e_{s}\right\| \bar{\rho}(A) \\
& -k_{v} \underline{\rho}\left(L+\beta I_{N}\right)\left\|e_{s}\right\|^{2}-\frac{k_{F}}{2}\|\tilde{\Phi}\|_{F}^{2}+\frac{k_{F}}{2}\|\Phi\|_{F}^{2} \\
\leq & -\left[k_{v} \underline{\rho}\left(L+\beta I_{N}\right)-\bar{\rho}\left(L+\beta I_{N}\right) \Theta_{M}\right]\left\|e_{s}\right\|^{2} \\
& -\frac{k_{F}}{2}\|\tilde{\Phi}\|_{F}^{2}+\frac{1}{4} \bar{\rho}\left(L+\beta I_{N}\right) \Theta_{M} \\
& +\|\tilde{\Phi}\|_{F} \phi_{M}\left\|e_{s}\right\| \bar{\rho}(A)+\frac{k_{F}}{2}\|\Phi\|_{F}^{2} \leq-\alpha V+C,
\end{aligned}
$$

where

$$
\begin{aligned}
& \alpha=2 \min \left\{k_{\nu} \underline{\rho}\left(L+\beta I_{N}\right)-\bar{\rho}\left(L+\beta I_{N}\right) \Theta_{M}, \frac{k_{F} / 2}{\bar{\rho}\left(\Gamma^{-1}\right)}\right\}, \\
& C=\frac{1}{4} \bar{\rho}\left(L+\beta I_{N}\right) \Theta_{M}+\|\tilde{\Phi}\|_{F} \phi_{M}\left\|e_{s}\right\| \bar{\rho}(A)+\frac{k_{F}}{2}\|\Phi\|_{F}^{2} .
\end{aligned}
$$

The control parameters are designed to satisfy

$$
k_{\nu} \underline{\rho}\left(L+\beta I_{N}\right)-\bar{\rho}\left(L+\beta I_{N}\right) \Theta_{M}>0 .
$$

According to (27), we have

$$
V(t) \leq V(0) e^{-\alpha t}+\frac{C}{\alpha}\left(1-e^{-\alpha t}\right) .
$$

Thus, the errors $e_{s}$ and $\tilde{\Phi}$ converge to compact sets $\Omega_{e_{s}}$ and $\Omega_{\tilde{\Phi}}$, which are defined as

$$
\begin{aligned}
& \Omega_{e_{s}}:=\left\{\left\|e_{s}\right\| \leq \sqrt{\frac{2 C}{\alpha}}\right\}, \\
& \Omega_{\tilde{\Phi}}:=\left\{\|\tilde{\Phi}\| \leq \sqrt{\frac{2 C}{\alpha \underline{\rho}\left(\Gamma^{-1}\right)}}\right\} .
\end{aligned}
$$

It can be seen from the above analysis that the errors $e_{s}$ and $\tilde{\Phi}$ are bounded. The system can achieve the stability rather than the exponential stability, and the boundedness can be adjusted by choosing proper control parameters. This completes the proof.

\section{Simulations}

In this section, three simulations are presented to support our theoretical analysis. Firstly, we will show that a formation in circular shape is changed into elliptical shape during the moving so as to pass a narrow space. The second simulation will verify that the remaining robots can reconstruct the formation when some robots fail or are lost due to some 


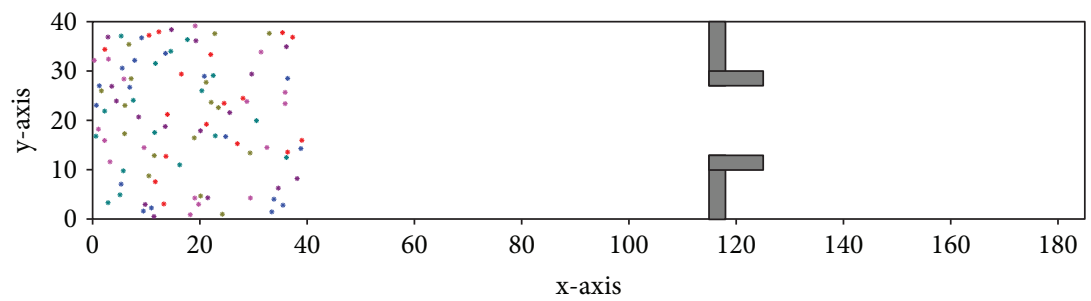

(a) Initial positions

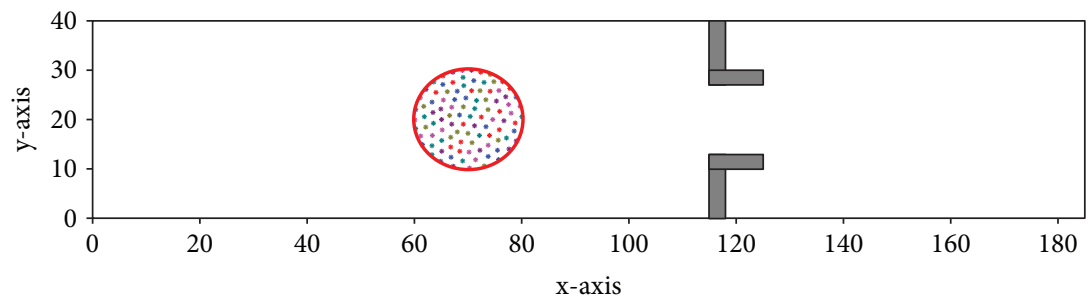

(b) Circular formation achievement

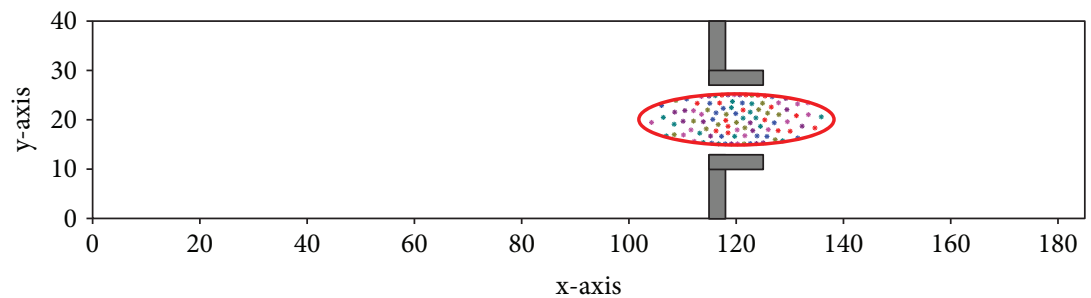

(c) Change to elliptical formation

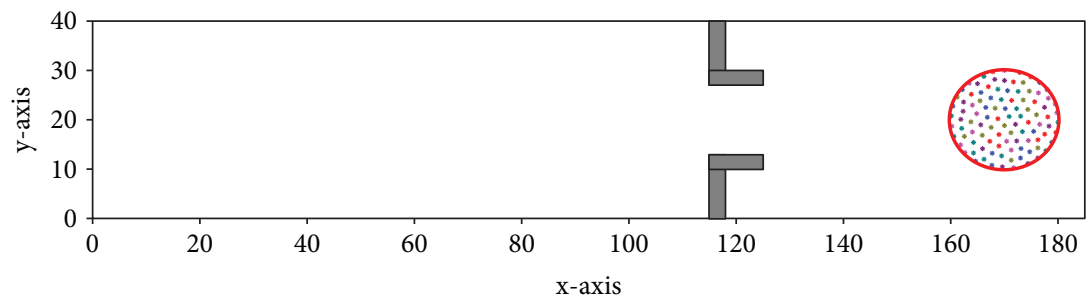

(d) Back to circular formation

FIGURE 1: Change of the formation shape for passing the narrow space.

accidents. Finally, two moving formations will be merged together by applying the proposed formation control law.

In the three simulations, 100 robots are considered with the positions initialized randomly in an area of $40 \mathrm{~m}$ long and $40 \mathrm{~m}$ wide and the velocities initialized randomly within $4 \mathrm{~m} / \mathrm{s}$ each direction. The basic control parameters are set as $k_{g}=1.4, k_{v}=1.3, \beta=0.8$, and $k_{q}=2$. The parameters for updating the RBFNN weights are $\gamma=1$ and $k_{F}=0.5$. The disturbances are set as $w_{i}(t)=\tau_{i}[0.1 \cos (5 t) \sin (6 t)+0.2 \sin$ $\left.(9 t), 0.1 \cos (3 t) \sin (6 t)+0.2(\cos (8 t))^{2}\right]^{T}$, where $\tau_{i}$ is a random number between 0 and 1 . The unknown nonlinear dynamics are defined as $f_{i}(t)=\left[0.02 v_{i x}^{2}+0.1 v_{i y}^{3}, 0.01 v_{i x}+\right.$ $\left.0.03 v_{i x}^{2} v_{i y}^{2}\right]^{T}$.

4.1. Passing a Narrow Space. In this section, the desired formation is a circular shape with the radius of $10 \mathrm{~m}$ and the velocity $v_{r}=[5,0] \mathrm{m} / \mathrm{s}$. It is shown in Figure $1(\mathrm{~b})$ that the swarm of robots achieved the circular formation at $t=10 \mathrm{~s}$.
Then, the swarm of robots changed its formation into the elliptical shape for passing a narrow space. The elliptical shape is set with the long radius of $18 \mathrm{~m}$ and the short radius of $5 \mathrm{~m}$. The elliptical formation in the narrow space is shown in Figure 1 (c) at $t=20 \mathrm{~s}$. After passing the narrow space, the swarm of robots changed back to the circular formation as illustrated in Figure 1(d) at $t=30 \mathrm{~s}$. Further, the robots can maintain the formation shape while moving.

4.2. Loss of Robots. In this section, the swarm of robots are supposed to achieve the circular formation as the same as that in Section 4.1. It is assumed that $2 / 3$ of the robots failed or are lost due to some accidents at $t=20 \mathrm{~s}$, as shown in Figure 2(c). Then, the remaining $1 / 3$ of the robots should continue to complete the task of region-based formation. It is shown in Figure 2(d) that the remaining robots reconstruct the circular formation again by adjusting the detection range of the robots adaptively. 


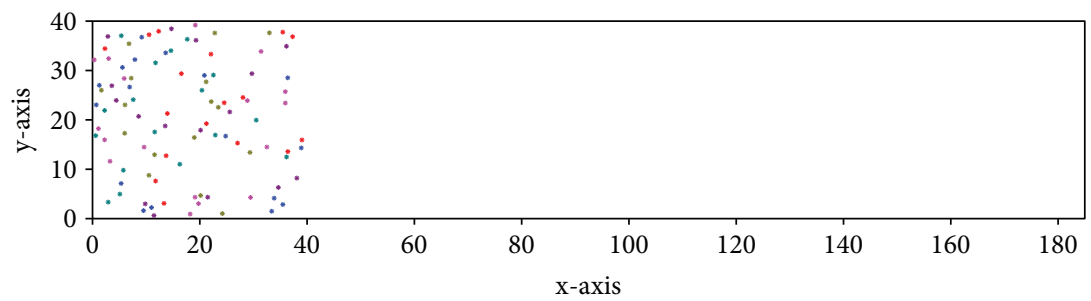

(a) Initial positions



(b) Circular formation achievement

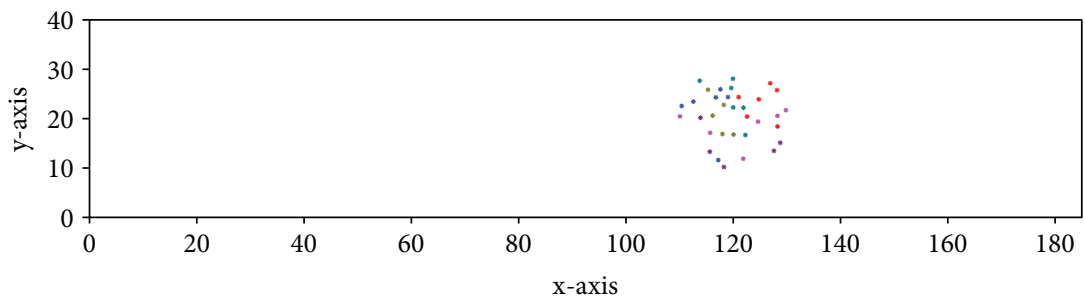

(c) The remaining robots

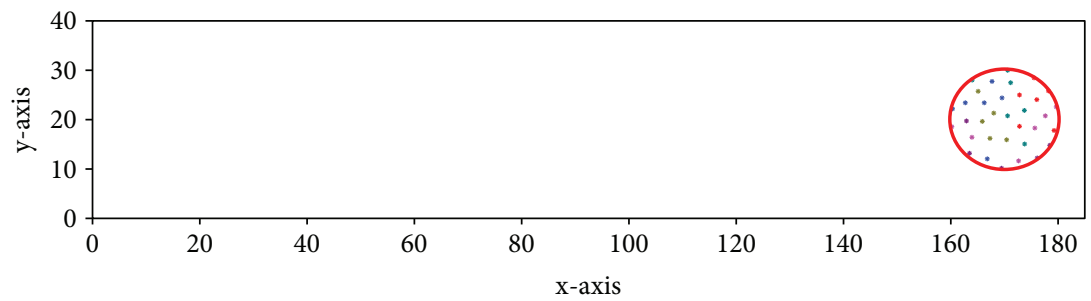

(d) Formation reconstruction

FIGURE 2: Formation reconstruction with the remaining robots.

4.3. Merging of Formations. The 80 robots are initialized randomly and shown as red dots, while the other 20 robots are initialized randomly and shown as blue dots in Figure 3(a). The red robots achieved the desired circular formation with the radius of $10 \mathrm{~m}$ and the velocity $v_{r}=[5,-0.1] \mathrm{m} / \mathrm{s}$. On the other hand, the blue robots achieved the circular formation with the radius of $5 \mathrm{~m}$ and the velocity $v_{r}=[5,0.13] \mathrm{m} /$ $s$, as shown in Figure 3(b). The two formations were getting closer to each other, and they started to merge at about $t=$ 21.7 s, as shown in Figure 3(c). Finally, the blue formation and the red formation merged together into one formation with the radius of $5 \sqrt{5} \mathrm{~m}$ and the velocity $v_{r}=[5,0] \mathrm{m} / \mathrm{s}$. It can be seen from Figure 3(d) that the density of the merged formation is the same as that of the original formations.

\section{Conclusion}

In this paper, we investigate a decentralized region-based formation control law for a swarm of robots. The unknown nonlinear dynamics of the robots are approximated by an adaptive neural network, and the desired formation shape is achieved by designing appropriate potential functions. The collision avoidance, velocity consensus, and region tracking are all considered in the controller. With the proposed formation control law, the formation can pass a narrow space by changing the shape of the formation. When some robots failed due to some accidents, the formation can be reconstructed adaptively with the remaining robots. In addition, the proposed region-based formation control scheme is effective in dealing with the formation merging problem.

In the future, we will design more flexible formation structure and more intelligent controllers based on the learning control method $[28,29]$. The adaptive observer and parameter estimation may be applied in the shape control $[30,31]$. In addition, the limited maneuverability and the sensor failure of the robot will be considered in our future work [32-34]. 


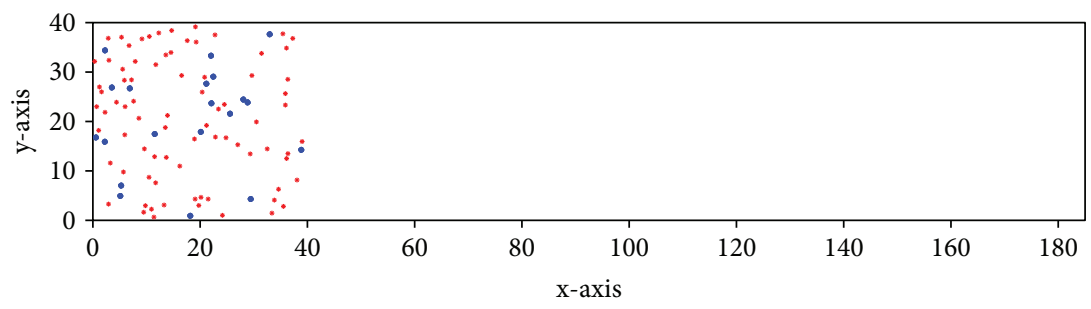

(a) Initial positions

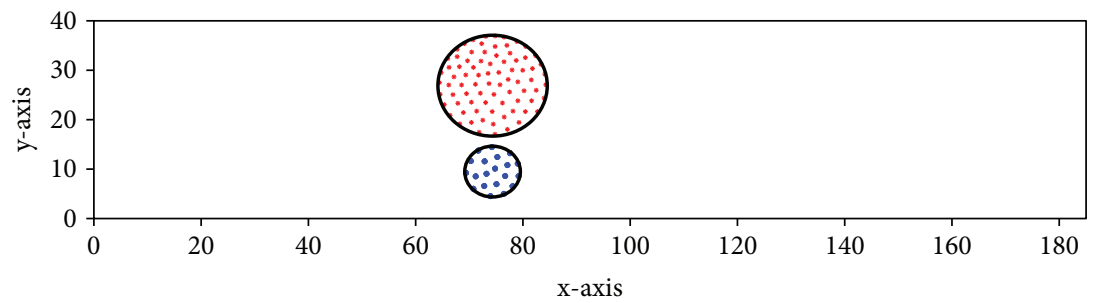

(b) Two separate formations



(c) The two formations are close to each other

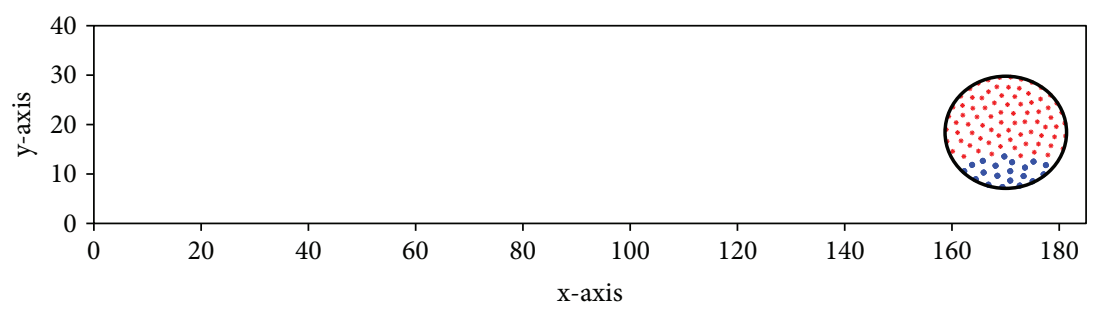

(d) Merge into one formation

FIgURE 3: Merging of the two formations.

\section{Data Availability}

The data used to support the findings of this study are included within the article, such as the basic control parameters, the parameter for updating the RBFNN weights, and the size and velocity of the desired region. Moreover, all data used in this study are available from the corresponding author upon request.

\section{Conflicts of Interest}

The authors declare that they have no conflicts of interest.

\section{Acknowledgments}

This work was supported by the National Natural Science Foundation of China (Grant nos. 61473124, 61803111, 61876047, and 11832009).

\section{References}

[1] L. Bayındır, "A review of swarm robotics tasks," Neurocomputing, vol. 172, pp. 292-321, 2016.

[2] H. Rezaee and F. Abdollahi, "A decentralized cooperative control scheme with obstacle avoidance for a team of mobile robots," IEEE Transactions on Industrial Electronics, vol. 61, no. 1, pp. 347-354, 2014.

[3] Y.-Y. Chen and Y.-P. Tian, "Formation tracking and attitude synchronization control of underactuated ships along closed orbits," International Journal of Robust and Nonlinear Control, vol. 25, no. 16, pp. 3023-3044, 2015.

[4] X. J. Lan, L. Liu, and Y. J. Wang, “Online trajectory planning and guidance for reusable launch vehicles in the terminal area," Acta Astronautica, vol. 118, pp. 237-245, 2016.

[5] X. J. Lan, Y. J. Wang, and L. Liu, "Dynamic decoupling tracking control for the polytopic LPV model of hypersonic vehicle," Science China Information Sciences, vol. 58, no. 9, pp. 1-14, 2015. 
[6] M. Deghat, B. D. O. Anderson, and Z. Lin, "Combined flocking and distance-based shape control of multi-agent formations," IEEE Transactions on Automatic Control, vol. 61, no. 7, pp. 1824-1837, 2016.

[7] H. Huang, C. Yu, and Q. Wu, "Autonomous scale control of multiagent formations with only shape constraints," International Journal of Robust and Nonlinear Control, vol. 23, no. 7, pp. 765-791, 2013.

[8] S. Li, J. Zhang, X. Li, F. Wang, X. Luo, and X. Guan, "Formation control of heterogeneous discrete-time nonlinear multiagent systems with uncertainties," IEEE Transactions on Industrial Electronics, vol. 64, no. 6, pp. 4730-4740, 2017.

[9] M. Aranda, G. Lopez-Nicolas, C. Sagues, and M. M. Zavlanos, "Distributed formation stabilization using relative position measurements in local coordinates," IEEE Transactions on Automatic Control, vol. 61, no. 12, pp. 3925-3935, 2016.

[10] Q. Liu, Z. Wang, X. He, and D. Zhou, "Event-based distributed filtering over Markovian switching topologies," IEEE Transactions on Automatic Control, 2018.

[11] X. Dong, J. Xi, G. Lu, and Y. Zhong, "Formation control for high-order linear time-invariant multiagent systems with time delays," IEEE Transactions on Control of Network Systems, vol. 1, no. 3, pp. 232-240, 2014.

[12] M. C. Park, K. Jeong, and H. S. Ahn, "Formation stabilization and resizing based on the control of inter-agent distances," International Journal of Robust and Nonlinear Control, vol. 25, no. 14, pp. 2532-2546, 2015.

[13] K. K. Oh and H. S. Ahn, "Leader-follower type distance-based formation control of a group of autonomous agents," International Journal of Control, Automation and Systems, vol. 15, no. 4, pp. 1738-1745, 2017.

[14] M. Aranda, G. López-Nicolás, C. Sagüés, and M. M. Zavlanos, "Coordinate-free formation stabilization based on relative position measurements," Automatica, vol. 57, pp. 11-20, 2015.

[15] H. Oh, A. Ramezan Shirazi, C. Sun, and Y. Jin, "Bio-inspired self-organising multi-robot pattern formation: a review," Robotics and Autonomous Systems, vol. 91, pp. 83-100, 2017.

[16] S. Sam Ge, J. Zhang, X. Cao, and X. Sun, "Region tracking control for high-order multi-agent systems in restricted space," IET Control Theory \& Applications, vol. 10, no. 4, pp. 396406, 2016.

[17] C. C. Cheah, S. P. Hou, and J. J. E. Slotine, "Region-based shape control for a swarm of robots," Automatica, vol. 45, no. 10 , pp. 2406-2411, 2009.

[18] H. Jung and D. H. Kim, "Potential-function-based shape formation in swarm simulation," International Journal of Control, Automation and Systems, vol. 12, no. 2, pp. 442-449, 2014.

[19] R. Haghighi and C. C. Cheah, "Multi-group coordination control for robot swarms," Automatica, vol. 48, no. 10, pp. 2526-2534, 2012.

[20] S. P. Hou and C. C. Cheah, "Dynamic compound shape control of robot swarm," IET Control Theory \& Applications, vol. 6, no. 3, pp. 454-460, 2012.

[21] S. P. Hou, C. C. Cheah, and J. J. E. Slotine, "Dynamic region following formation control for a swarm of robots," in 2009 IEEE International Conference on Robotics and Automation, pp. 1528-1533, Kobe, Japan, 2009.

[22] J. M. Hendrickx, C. Yu, B. Fidan, and B. D. O. Anderson, "Rigidity and persistence for ensuring shape maintenance of multi-agent meta-formations," Asian Journal of Control, vol. 10, no. 2, pp. 131-143, 2008.
[23] C. Yu, B. Fidan, and B. D. O. Anderson, "Principles to control autonomous formation merging," in 2006 American Control Conference, p. 7, Minneapolis, MN, USA, 2006, IEEE.

[24] T. Han, Z. Lin, and M. Fu, "Three-dimensional formation merging control under directed and switching topologies," Automatica, vol. 58, pp. 99-105, 2015.

[25] Z. Zhao, X. Wang, C. Zhang, Z. Liu, and J. Yang, "Neural network based boundary control of a vibrating string system with input deadzone," Neurocomputing, vol. 275, pp. 10211027, 2018.

[26] Z. Zhao, J. Shi, X. Lan, X. Wang, and J. Yang, "Adaptive neural network control of a flexible string system with nonsymmetric dead-zone and output constraint," Neurocomputing, vol. 283, pp. 1-8, 2018.

[27] C. Yang, X. Wang, Z. Li, Y. Li, and C. Y. Su, "Teleoperation control based on combination of wave variable and neural networks," IEEE Transactions on Systems, Man, and Cybernetics: Systems, vol. 47, no. 8, pp. 2125-2136, 2017.

[28] B. Xu and F. Sun, "Composite intelligent learning control of strict-feedback systems with disturbance," IEEE Transactions on Cybernetics, vol. 48, no. 2, pp. 730-741, 2018.

[29] S. L. Dai, M. Wang, and C. Wang, "Neural learning control of marine surface vessels with guaranteed transient tracking performance," IEEE Transactions on Industrial Electronics, vol. 63, no. 3, pp. 1717-1727, 2016.

[30] C. Yang, Y. Jiang, W. He, J. Na, Z. Li, and B. Xu, "Adaptive parameter estimation and control design for robot manipulators with finite-time convergence," IEEE Transactions on Industrial Electronics, vol. 65, no. 10, pp. 8112-8123, 2018.

[31] C. Yang, K. Huang, H. Cheng, Y. Li, and C. Y. Su, "Haptic identification by ELM-controlled uncertain manipulator," IEEE Transactions on Systems, Man, and Cybernetics: Systems, vol. 47, no. 8, pp. 2398-2409, 2017.

[32] Q. Liu, Z. Wang, X. He, and D. H. Zhou, "Event-triggered resilient filtering with measurement quantization and random sensor failures: monotonicity and convergence," Automatica, vol. 94, pp. 458-464, 2018.

[33] J. Huang, Y. Wang, and T. Fukuda, "Set-membership-based fault detection and isolation for robotic assembly of electrical connectors," IEEE Transactions on Automation Science and Engineering, vol. 15, no. 1, pp. 160-171, 2018.

[34] Q. Liu, Z. Wang, X. He, and D. H. Zhou, "On Kalmanconsensus filtering with random link failures over sensor networks," IEEE Transactions on Automatic Control, vol. 63, no. 8, pp. 2701-2708, 2018. 


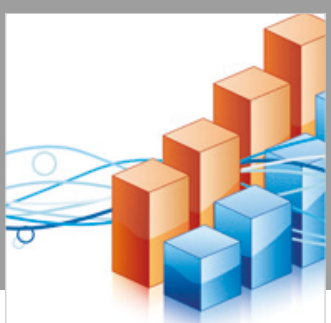

Advances in

Operations Research

\section{-n-m}
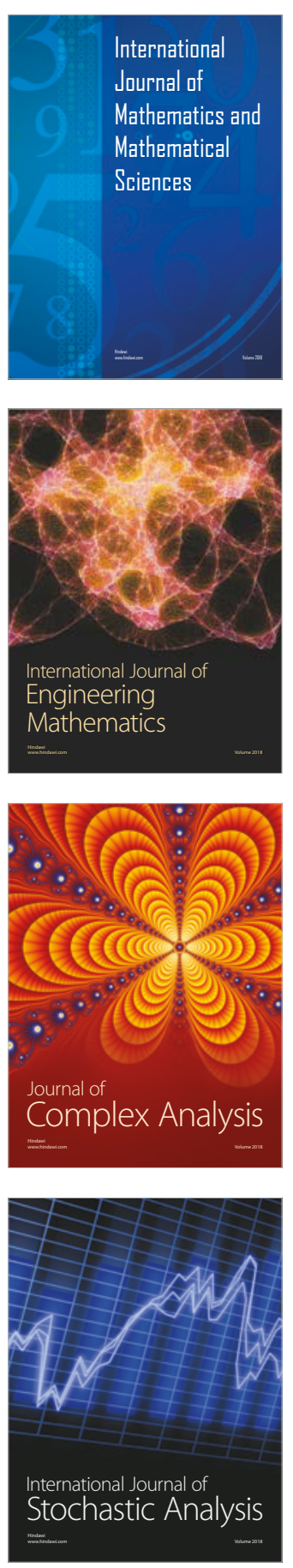
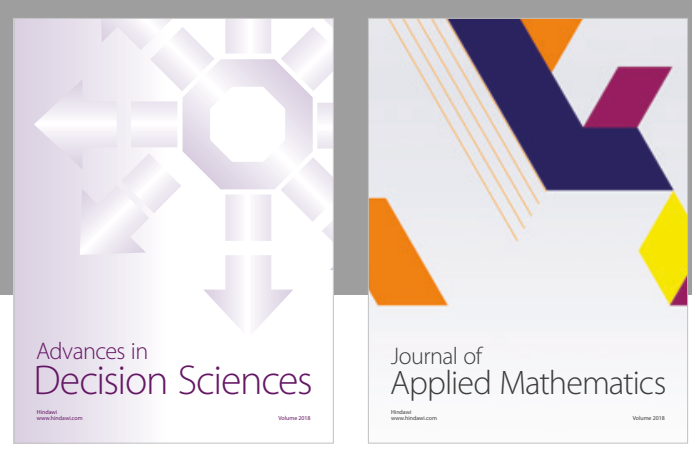

Journal of

Applied Mathematics
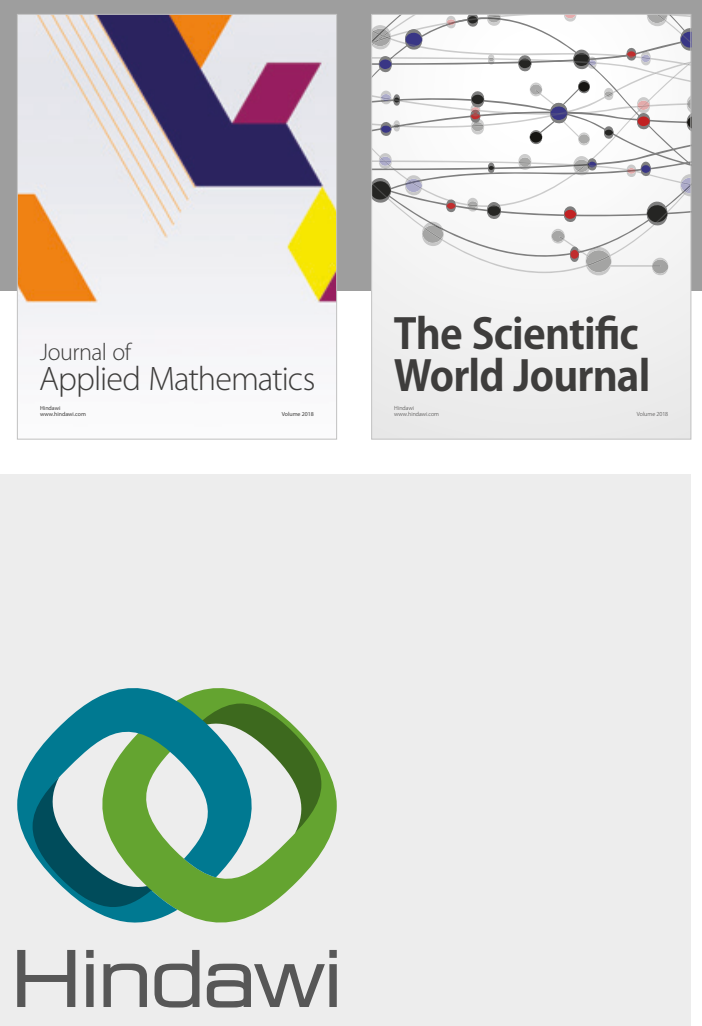

Submit your manuscripts at

www.hindawi.com

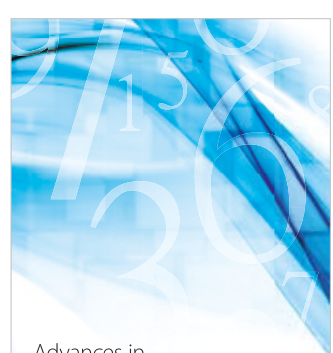

Advances in
Numerical Analysis
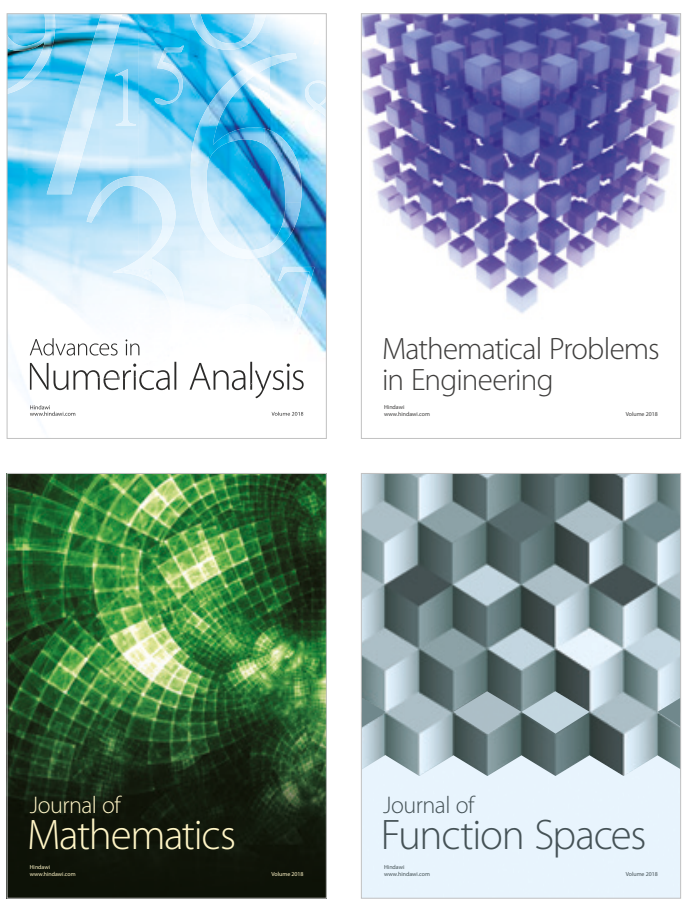

Mathematical Problems in Engineering



International Journal of

Differential Equations

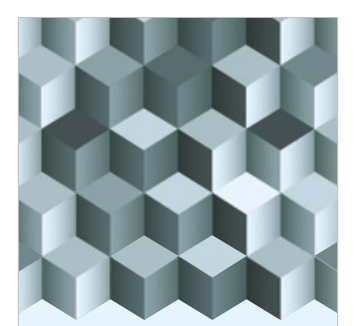

Journal of

Function Spaces
The Scientific

World Journal

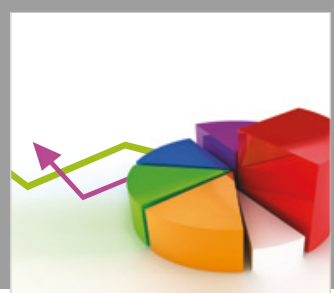

Journal of

Probability and Statistics
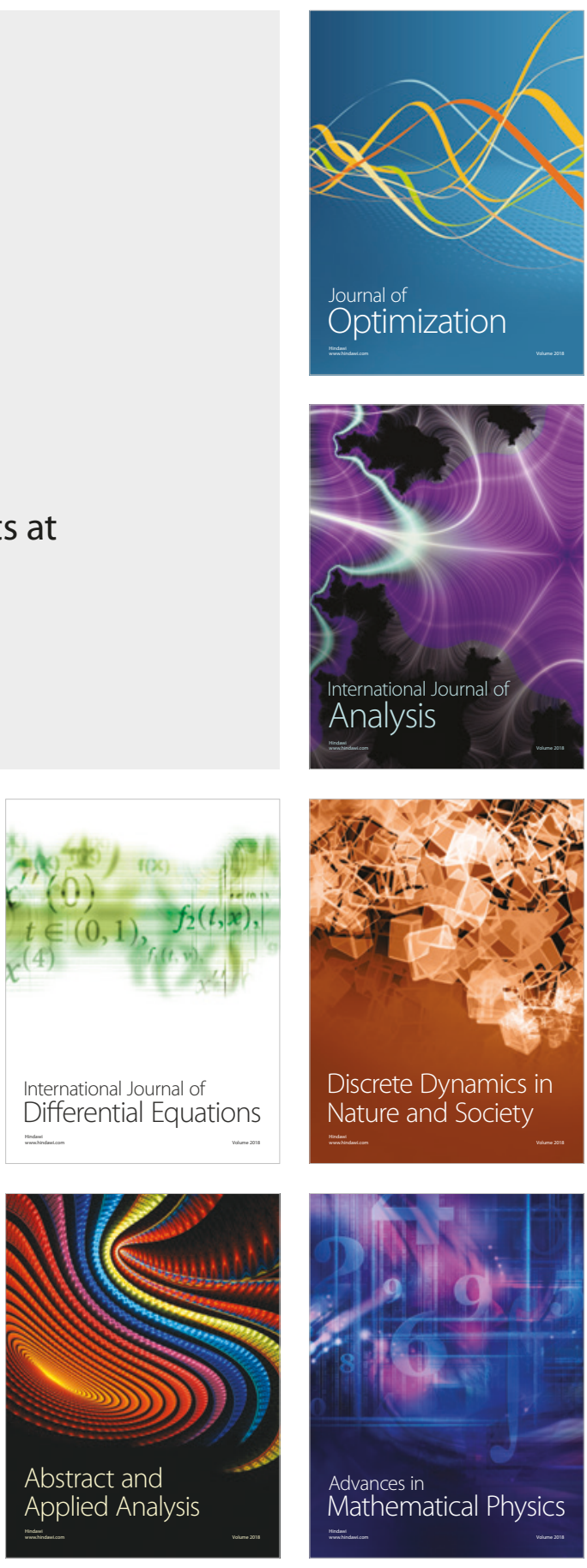